
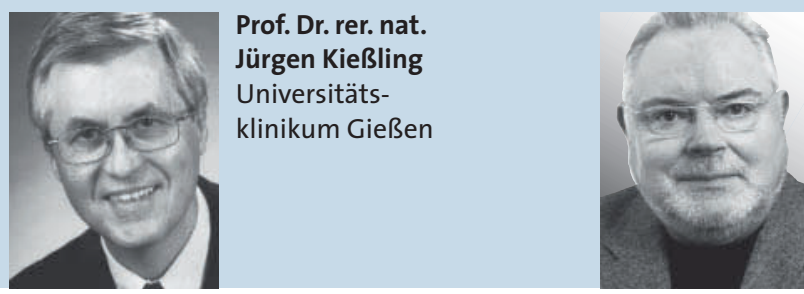

Dr. med.

Dieter Leithäuser

HNO-Arzt, Warburg

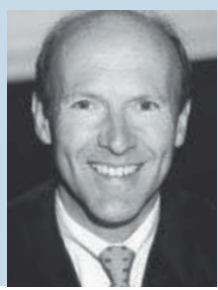

Prof. Dr. med.

Ralph Mösges

Universität Köln

\section{Spezifische Immuntherapie funktioniert auch epikutan}

\author{
Neben dem Subkutangewebe und der Mundschleimhaut bietet sich \\ die Haut als Zielorgan für die spezifische Immuntherapie an. Die Epidermis \\ verfügt mit ihren Langerhans-Zellen über ein dichtes Netz Antigen- \\ präsentierender Zellen, ist aber nicht vaskularisiert und macht die \\ epikutane Immuntherapie damit zu einem potenziell sicheren Therapie- \\ verfahren mit wenig systemischen Nebenwirkungen.
}

$\mathrm{n}$ einer Pilotstudie sollten erste klinische Daten zur perkutanen Immuntherapie gesammelt werden. Einbezogen in die monozentrische Phase I/II-Studie waren 37 Patienten mit einer Gräserpollen-Rhinitis, die randomisiert und doppelblind entweder ein Allergenpflaster (n = 21) oder ein Placebo-Pflaster $(\mathrm{n}=16)$ erhielten. Eingesetzt wurde ein Pflastersystem, das von der Krankenhausapotheke des Universitätskrankenhauses in Zürich entwickelt und hergestellt wurde. Das selbstklebende Pflaster enthält ein mit Vaseline gefülltes Reservoir, in die pro Pflaster 300 IR Phleum pratense-Extrakt der Firma Stallergenes eingearbeitet ist. Das Pflaster bleibt für 48 Stunden auf der Haut und gibt die Allergene kontinuierlich in die Epidermis ab. Die Behandlung erfolgte prä- und kosaisonal über zwei Jahre, primäres Studienziel waren Veränderungen im nasalen Provokationstest jeweils nach Abschluss eines Behandlungszyklus.

Bei den mit dem allergenhaltigen Pflaster behandelten Patienten gingen die Scores im nasalen Provokationstest im ersten und zweiten Jahr signifikant zurück ( $p<0,001$ im ersten Jahr, $p=0,003$ im zweiten Jahr), unter Placebo war im ersten Jahr ebenfalls ein Rückgang des Scores zu verzeichnen, im zweiten Jahr blieb dieser Effekt aus ( $\mathrm{p}=0,03$ und $\mathrm{p}=$ 0,53). Der Vergleich zwischen den beiden Gruppen ergab bei den Provokationstest-Scores keine Unterschiede, der Gesamtbehandlungserfolg wurde von den Patienten der Verumgruppe signifikant besser beurteilt als von den Patienten der Placebogruppe.

Die Verträglichkeit war insgesamt gut. Bei Patienten der Verumgruppe traten allerdings häufig leichte Ekzeme an der Applikationsstelle auf - eine Nebenwirkung, die wahrscheinlich durch stimulierte allergenspezifischen T-Zellen hervorgerufen wird.

bk

Senti G et al. Epicutaneous allergen administration as a novel method of allergenspecific immunotherapy. J Allergy Clin

Immunol 2009;124:997-1002

\section{Kommentar}

Dies ist die erste klinische doppelblinde placebokontrollierte Studie, welche die Wirksamkeit einer epikutanen spezifischen Immuntherapie (SIT) an einem größeren Kollektiv von Patienten mit einer allergischen Rhinitis untersucht hat. Dieser innovative Ansatz ist zweifelsohne interessant. Allerdings werden die Ergebnisse dieser Studie limitiert durch einige methodische Schwä- chen im Studiendesign. So wurde nur das erste Pflaster in der Klinik von den Studienärzten appliziert, die weiteren Pflaster wurden von den Patienten zu Hause selbst aufgebracht. Aufgrund des Auftretens von lokalen (leichtgradigen) Ekzemen in der Verumgruppe darf angenommen werden, dass dies zur Entblindung eines Teils der Patienten geführt hat (ein methodisches Problem in einigen Studien zur SIT). Ferner wurde der Gesamtbehandlungserfolg der Therapie durch die Patienten retrospektiv im Vergleich zu den Vorjahren beurteilt, was die Interpretation dieser Resultate wegen eines gewissen „recall-bias“ einschränkt. Der primäre Endpunkt dieser Studie, die Scores im NPT, zeigte darüber hinaus im Vergleich der beiden Gruppen keine signifikanten Unterschiede. Dieses Ergebnis könnte sich durch die relativ geringe Anzahl von Studienpatienten $(n=37)$ erklären lassen. Auch immunologische Parameter wurden nicht monitoriert.

Zusammenfassend untersuchte die publizierte „proof-of-concept“-Studie eine sehr interessante, innovative Form der SIT. Allerdings wird eine weiterführende Beurteilung der klinischen und immunologischen Effekte der epikutanen Form der Therapie erst durch umfangreichere Phase-III-Studien mit einer höheren Anzahl von Patienten und einem modifizierten Studiendesign möglich sein.

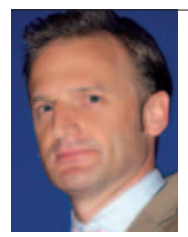

Dr. med. Oliver Pfaar Zentrum für Rhinologie und Allergologie Wiesbaden der HNO-Universitätsklinik Mannheim 